\title{
Propositional Dynamic Logic for Concurrent Programs
}

\author{
By \\ Hirokazu NishimuRA*
}

\begin{abstract}
We give a nice Gentzen-type axiomatization of propositional dynamic logic (PDL) with the shuffle opcrator $\alpha / / \beta$ (cobegin...coend)
\end{abstract}

\section{$\S 1$. Introduction}

One of the most significant topics that were recommended by Harel [3] for further research is to develop a theoretical framework for concurrent programs in the spirit of his thesis for sequential programs. To this end, several challenging subjects must be attacked vigorously. For one thing, we have to develop a powerful machinery which can deal with rich temporal properties of concurrent programs. Complex interactions of concurrent processes require formal analysis of what a program does while it is running, whereas partial and total correctness are the only important properties of sequential programs. Such formal machineries are now being developed under the name of process logic, notably Harel, Kozen and Parikh [4], Nishimura [6], Pratt [9], etc. For another thing, a nice axiomatization of PDL with the shuffle operator $\alpha / / \beta$ must be provided, which we are about to do in this paper. For its first-order version, the reader can consult Nishimura [7].

\section{§. Concurrent Propositional Dynamic Logic}

The main purpose of this section is to present the exact syntax and semantics of concurrent propositional dynamic logic (CPDL). First of all, we define the notion of a program $\alpha$ by induction as follows:

Received, February 20, 1981.

* Research Institute for Mathematical Sciences, Kyoto Univcrsity, Kyoto 606, Japan. 
(1) The program constant $\theta$ (a program for "do nothing") is a program.

(2) Each atomic program, denoted by a, b, c,.., is a program.

(3) If $\alpha$ and $\beta$ are programs, so too are $\alpha ; \beta, \alpha \cup \beta, \alpha^{* *}$ and $\alpha / / \beta$.

Programs of the form indicated in (1) and (2) are called indivisible programs.

The notion of a wff $A$ is defined by induction as follows:

(1) Each atomic formula, denoted by $p, q, \ldots$, is a wff.

(2) If $A$ and $B$ are wffs, so too are $A \wedge B, A \vee B, \neg A$ and $A \supset B$.

(3) If $A$ is a wff and $\alpha$ is a program, then $[\alpha] A$ is a wff.

In this paper we do not include programs of the form $A$ ? and $\alpha^{-}$for the sake of simplicity.

Let $\mathbf{N}$ be the set of all nonnegative integers. We define a function $\varphi$ from $\mathbb{N} \times \mathbb{N}$ to $\mathbb{N}$ as follows:

$$
\varphi(i, j)=\frac{(i+j+1)(i+j)}{2}+j \quad \text { for each } \quad(i, j) \in \mathbb{N} \times \mathbb{N} .
$$

We know well in elementary mathematics that $\varphi$ is a one-to-one correspondence of $\mathbb{N} \times \mathbb{N}$ onto $\mathbb{N}$ and if $i+j<i^{\prime}+j^{\prime}$, then $\varphi(i, j)<\varphi\left(i^{\prime}, j^{\prime}\right)$. We denote by $\psi$ and $\chi$ the functions from $N$ to $N$ satisfying the following condition:

$$
\varphi(\psi(i), \chi(i))=i \quad \text { for any } \quad i \in \mathbb{N} .
$$

We define a function $d$ from the set of all programs to $\mathbb{N}$ as follows:

(1) $\mathrm{d}(\alpha)=1$ for any indivisible program $\alpha$.

(2) $\mathrm{d}(\alpha ; \beta)=\mathrm{d}(\alpha \cup \beta)=2(\max \{\mathrm{d}(\alpha), \mathrm{d}(\beta)\}+1)$.

(3) $\mathrm{d}\left(\alpha^{*}\right)=\mathrm{d}(\alpha)+1$.

(4) $\mathrm{d}(\alpha / / \beta)=\varphi(\mathrm{d}(\alpha), \mathrm{d}(\beta))+1$.

We define two functions init and rest from $N \times P M$ to $P M$, where $P M$ is the set of all programs, as follows:

(0) $\quad$ init $(0, \alpha)=\theta$ and rest $(0, \alpha)=\alpha$ for any program $\alpha$.

(1) init $(i+1, \alpha)=\alpha$ and rest $(i+1, \alpha)=\theta$ for any indivisible program $\alpha$.

(2a) $\operatorname{init}(2 i+1, \alpha ; \beta)=\operatorname{init}(i, \alpha)$ and $\operatorname{rest}(2 i+1, \alpha ; \beta)=\operatorname{rest}(i, \alpha) ; \beta$.

(2b) $\quad$ init $(2(i+1), \alpha ; \beta)=\alpha$; init $(i, \beta)$ and rest $(2(i+1), \alpha ; \beta)=\operatorname{rest}(i, \beta)$.

(3a) init $(2 i+1, \alpha \cup \beta)=\operatorname{init}(i, \alpha)$ and $\operatorname{rest}(2 i+1, \alpha \cup \beta)=\operatorname{rest}(i, \alpha)$.

(3b) init $(2(i+1), \alpha \cup \beta)=\operatorname{init}(i, \beta)$ and $\operatorname{rest}(2(i+1), \alpha \cup \beta)=\operatorname{rest}(i, \beta)$.

(4) $\quad$ init $(i+1, \alpha / / \beta)=\operatorname{init}(\psi(i), \alpha) / /$ init $(\chi(i), \beta)$ and rest $(i+1, \alpha / / \beta)=$ $\operatorname{rest}(\psi(i), \alpha) / / \operatorname{rest}(\chi(i), \beta)$.

(5) $\quad \operatorname{init}\left(i+1, \alpha^{*}\right)=\alpha^{*}$; init $(i, \alpha)$ and rest $\left(i+1, \alpha^{*}\right)=\operatorname{rest}(i, \alpha) ; \alpha^{*}$. 
We define the rest-closure of a program $\alpha$, written rest-cl( $\alpha)$, as the set

$$
\{\operatorname{rest}(i, \alpha) \mid 0 \leqq i \leqq \mathrm{~d}(\alpha)\}
$$

We recall the following proposition of Nishimura [7].

Proposition 2.1. For any program $\alpha$, any $\beta \in \operatorname{rest-cl}(\alpha)$ and any $i \in \mathbb{N}$, we have $\operatorname{rest}(i, \beta) \in \operatorname{rest}-\mathrm{cl}(\alpha)$. I.e., rest-cl $(\alpha)$ is closed with respect to the operation rest.

We give some examples of rest-cl $(\alpha)$.

(1) $\operatorname{rest-cl}(\theta)=\{\theta\}$.

(2) $\operatorname{rest-cl}(a ; b)=\{a ; b, \theta ; b, b, \theta\}$.

(3) $\operatorname{rest-cl}\left((a ; b)^{*}\right)=\left\{(a ; b) ;(a ; b)^{*},(\theta ; b) ;(a ; b)^{*}, b ;(a ; b)^{*}, \theta ;(a ; b)^{*}\right\}$.

(2) and (3) are completely precise but look somewhat superfluous. The following simplified versions are much clearer.

$\left(2^{\prime}\right) \operatorname{rest-cl}(a ; b)=\{a ; b, b, \theta\}$.

$\left(3^{\prime}\right) \operatorname{rest}-\mathrm{cl}\left((a ; b)^{*}\right)=\left\{(a ; b)^{*}, b ;(a ; b)^{*}\right\}$.

We now turn our attention to semantics. A structure is a triple $(W, \rho, \pi)$, where

(1) $W$ is a nonempty set (heuristically, of possible states).

(2) $\rho$ is a function from the set of all programs to $2^{W \times W}$.

(3) $\pi$ is a function from $A F \times W$ to $\{0,1\}$, where $A F$ is the set of all atomic formulas. I.e., $\pi(p, w)$ denotes the truth value of $p$ at $w$.

An ordered pair $(w, v)$ of states $w$ and $v$ in $W$ is called a move. A finite sequence $\left(w_{1}, v_{1}\right) \cdots\left(w_{n}, v_{n}\right)$ of moves is called a path, while $w_{1}$ and $v_{n}$ are called the initial and final states of the path respectively. We denote by $\mathrm{H}(W)$ the set of all paths in $W$. For any $h \in \mathrm{H}(W)$, we denote the initial and final states of $h$ by is $(h)$ and $\mathrm{fs}(h)$ respectively. A path $\left(w_{1}, v_{1}\right) \cdots\left(w_{n}, v_{n}\right)$ is called legal if $v_{i}=w_{i+1}$ for each $1 \leqq i \leqq n-1$. We denote by $\mathrm{H}_{r}(W)$ the set of all legal paths. Given two subsets $S$ and $T$ of $\mathrm{H}(W)$, we define:

(1) $S$; $T$ is the set of all concatenations of $h_{1} \in S$ followed by $h_{2} \in T$.

(2) $S^{*}$ is the least subset of $\mathrm{H}(W)$ which contains $S$ and $\{(w, w) \mid w \in W\}$ and which is closed under concatenation.

(3) $S / / T$ is the set of all interleaving sequences of $h_{1} \in S$ and $h_{2} \in T$.

Now we are ready to extend $\rho$ to all programs.

(1) $\rho(\theta)=W \times W$.

(2) $\rho(\alpha ; \beta)=\rho(\alpha) ; \rho(\beta)$. 
(3) $\rho(\alpha \cup \beta)=\rho(\alpha) \cup \rho(\beta)$.

(4) $\rho\left(\alpha^{*}\right)=\rho(\alpha)^{*}$.

(5) $\rho(\alpha / / \beta)=\rho(\alpha) / / \rho(\beta)$.

Let

$$
\rho_{r}(\alpha)=\rho(\alpha) \cap \mathrm{H}_{r}(W) .
$$

Now we are ready to extend $\pi$ to all wffs.

(1) $\pi(A \wedge B, w)=1$ iff $\pi(A, w)=1$ and $\pi(B, w)=1$.

(2) $\pi(A \vee B, w)=1$ iff $\pi(A, w)=1$ or $\pi(B, w)=1$.

(3) $\pi(\neg A, w)=1$ iff $\pi(A, w)=0$.

(4) $\pi(A \supset B, w)=1$ iff $\pi(A, w)=1$ implies $\pi(B, w)=1$.

(5) $\pi([\alpha] A, w)=1$ iff for any $h \in \rho_{r}(\alpha)$, is $(h)=w$ implies that $\pi(A$, fs $(h))=1$.

A sequent is an ordered pair $(\Gamma, \Delta)$ of finite sets of wffs, which we will usually denote by $\Gamma \rightarrow \Delta$. $\pi$ can be extended to all sequents as follows:

(1) $\pi(\Gamma \rightarrow \Delta, w)=1$ if for any $A \in \Gamma \pi(A, w)=1$ and for any $B \in \Delta \pi(B, w)=0$,

(2) $\pi(\Gamma \rightarrow \Delta, w)=0$ otherwise.

We say that a sequent $\Gamma \rightarrow \Delta$ is realizable if for some structure $(W, \rho, \pi)$ and some $w \in W, \pi(\Gamma \rightarrow \Delta, w)=1$. A sequent $\Gamma \rightarrow \Delta$ which is not realizable is called valid (notation: $\vDash \Gamma \rightarrow \Delta$ ).

\section{§3. Axiomatization}

The main purpose of this section is to present a Gentzen-type sequential axiomatization of CPDL, say, GCPDL, which consists of the following axioms and inference rules:

Axioms: $A \rightarrow A$

Rules: $\frac{\Gamma \rightarrow \Delta}{\Pi, \Gamma \rightarrow \Delta, \Sigma} \quad$ (extension)

$$
\begin{array}{ll}
\left.\begin{array}{l}
\frac{\Gamma \rightarrow \Delta, A \quad A, \Pi \rightarrow \Sigma}{\Gamma, \Pi \rightarrow \Delta, \Sigma} \quad \text { (cut) } \\
\frac{\Gamma \rightarrow \Delta, A \quad \Gamma \rightarrow \Delta, B}{\Gamma \rightarrow \Delta, A \wedge B} \quad(\rightarrow \wedge) \\
\frac{A, \Gamma \rightarrow \Delta}{A \wedge B, \Gamma \rightarrow \Delta} \\
\frac{B, \Gamma \rightarrow \Delta}{A \wedge B, \Gamma \rightarrow \Delta}
\end{array}\right\}(\wedge \rightarrow)
\end{array}
$$




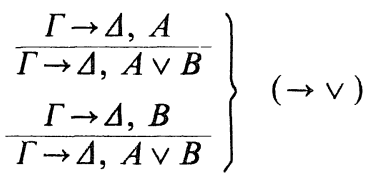

$$
\begin{aligned}
& \frac{A, \Gamma \rightarrow \Delta \quad B, \Gamma \rightarrow \Delta}{A \vee B, \Gamma \rightarrow \Delta} \quad(\vee \rightarrow) \\
& \frac{A, \Gamma \rightarrow \Delta}{\Gamma \rightarrow \Delta,\urcorner A} \quad(\rightarrow \neg)
\end{aligned}
$$

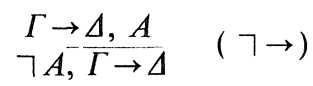

$$
\begin{aligned}
& \frac{A, \Gamma \rightarrow \Delta, B}{\Gamma \rightarrow \Delta, A \supset B} \quad(\rightarrow \supset) \\
& \stackrel{\Gamma \rightarrow \Delta, A \quad B, \Pi \rightarrow \Sigma}{A \supset B, \Gamma, \Pi \rightarrow \Delta, \Sigma} \quad(\supset \rightarrow) \\
& \frac{\Gamma \rightarrow A}{[\alpha] \Gamma \rightarrow[\alpha] A} \quad(\rightarrow[]) \\
& \frac{\Gamma \rightarrow \Delta, A}{\Gamma \rightarrow \Delta,[\theta] A} \quad(\rightarrow[\theta]) \\
& \frac{A, \Gamma \rightarrow \Delta}{[\theta] A, \Gamma \rightarrow \Delta} \quad([\theta] \rightarrow) \\
& \frac{\Gamma \rightarrow \Delta,[\alpha][\beta] A}{\Gamma \rightarrow \Delta,[\alpha ; \beta] A} \quad(\rightarrow[;]) \\
& \underset{[\alpha ; \beta] A, \Gamma \rightarrow \Delta}{[\alpha][\beta], \Gamma \rightarrow \Delta} \quad([;] \rightarrow) \\
& \frac{\Gamma \rightarrow \Delta,[\alpha] A \quad \Gamma \rightarrow \Delta,[\beta] A}{\Gamma \rightarrow \Delta,[\alpha \cup \beta] A} \quad(\rightarrow[\cup]) \\
& \left.\begin{array}{c}
\frac{[\alpha] A, \Gamma \rightarrow \Delta}{[\alpha \cup \beta] A, \Gamma \rightarrow \Delta} \\
\frac{[\beta] A, \Gamma \rightarrow \Delta}{[\alpha \cup \beta] A, \Gamma \rightarrow \Delta}
\end{array}\right\} \quad([\cup] \rightarrow) \\
& \frac{A \rightarrow C \quad C \rightarrow[\alpha] C \quad C \rightarrow B}{A \rightarrow\left[\alpha^{*}\right] B} \quad(\rightarrow[*]) \\
& \left.\begin{array}{c}
\frac{A, \Gamma \rightarrow \Delta}{\left[\alpha^{*}\right] A, \Gamma \rightarrow \Delta} \\
\frac{[\alpha]\left[\alpha^{*}\right] A, \Gamma \rightarrow \Delta}{\left[\alpha^{*}\right] A, \Gamma \rightarrow \Delta}
\end{array}\right\} \quad([*] \rightarrow) \\
& \frac{X}{\Gamma \rightarrow \Delta,[\alpha / / \beta] A} \quad(\rightarrow[/ /]),
\end{aligned}
$$

where $X=\{\Gamma \rightarrow \Delta$, [init $(i, \beta)][\alpha][\operatorname{rest}(i, \beta)] A \mid 0 \leqq i \leqq \mathrm{~d}(\beta)\}$ and $\alpha$ is an indivisible program. 


$$
\frac{[\operatorname{init}(i, \beta)][\alpha][\operatorname{rest}(i, \beta)] A, \Gamma \rightarrow \Delta}{[\alpha / / \beta] A, \Gamma \rightarrow \Delta} \quad([/ /] \rightarrow),
$$

where $\alpha$ is an indivisible program.

$$
\frac{X}{\Gamma \rightarrow \Delta,[(\alpha ; \beta) / / \gamma] A} \quad(\rightarrow[(;) / /]),
$$

where $X=\{\Gamma \rightarrow \Delta,[\alpha / / \operatorname{init}(i, \gamma)][\beta / / \operatorname{rest}(i, \gamma)] A \mid 0 \leqq i \leqq \mathrm{~d}(\gamma)\}$.

$$
\begin{aligned}
& \frac{[\alpha / / \operatorname{init}(i, \gamma)][\beta / / \operatorname{rest}(i, \gamma)] A, \Gamma \rightarrow \Delta}{[(\alpha ; \beta) / / \gamma] A, \Gamma \rightarrow \Delta} \quad([(;) / /] \rightarrow) \\
& \frac{\Gamma \rightarrow \Delta,[\alpha / / \gamma] A \quad \Gamma \rightarrow \Delta,[\beta / / \gamma] A}{\Gamma \rightarrow \Delta,[(\alpha \cup \beta) / / \gamma] A} \quad(\rightarrow[(\cup) / /]) \\
& \left.\begin{array}{c}
\frac{[\alpha / / \gamma] A, \Gamma \rightarrow \Delta}{[(\alpha \cup \beta) / / \gamma] A, \Gamma \rightarrow \Delta} \\
\frac{[\beta / / \gamma] A, \Gamma \rightarrow \Delta}{[(\alpha \cup \beta) / / \gamma] A, \Gamma \rightarrow \Delta}
\end{array}\right\} \quad([(\cup) / /] \rightarrow) \\
& \frac{A \rightarrow C_{\beta} X_{1} X_{2}}{A \rightarrow\left[\alpha^{*} / / \beta\right] B} \quad(\rightarrow[(*) / /]),
\end{aligned}
$$

where $C_{\gamma}$ is a wff for each $\gamma \in \operatorname{rest}-\operatorname{cl}(\beta), X_{1}=\left\{C_{\gamma} \rightarrow[\alpha / /\right.$ init $(i, \gamma)] C_{\text {rest }(i, \gamma)} \mid$ $\gamma \in \operatorname{rest}-\operatorname{cl}(\beta)$ and $0 \leqq i \leqq \mathrm{~d}(\gamma)\}$ and $X_{2}=\left\{C_{\gamma} \rightarrow[\gamma] B \mid \gamma \in \operatorname{rest}-\operatorname{cl}(\beta)\right\}$.

$$
\left.\begin{array}{l}
\frac{[\beta] A, \Gamma \rightarrow \Delta}{\left[\alpha^{*} / / \beta\right] A, \Gamma \rightarrow \Delta} \\
\frac{[\alpha / / \text { init }(i, \beta)]\left[\alpha^{*} / / \text { rest }(i, \beta)\right] A, \Gamma \rightarrow \Delta}{\left[\alpha^{*} / / \beta\right] A, \Gamma \rightarrow \Delta}
\end{array}\right\} \quad([(*) / /] \rightarrow)
$$

A proof $P$ (in $\mathrm{GCPDL}$ ) is a tree of sequents satisfying the following conditions:

(1) The topmost sequents of $P$ are axiom sequents.

(2) Every sequent in $P$ except the lowest one is an upper sequent of an inference rule whose lower sequent is also in $P$.

A sequent $\Gamma \rightarrow \Delta$ is said to be provable (in $\mathrm{GCPDL}$ ) if there exists a proof whose lowest sequent is $\Gamma \rightarrow \Delta$. If a sequent $\Gamma \rightarrow \Delta$ is provable, then we write $\vdash \Gamma \rightarrow \Delta$ (in GCPDL). A sequent $\Gamma \rightarrow \Delta$ which is not provable is said to be consistent (in GCPDL).

It is a great pleasure to note that there exists a strong analogy, e.g., between 
rules $(\rightarrow[U])$ and $(\rightarrow[(U) / /])$. Indeed, if we take $\gamma$ to be $\theta$ and identify, e.g., $\alpha / / \theta$ with $\alpha$, then the rule $(\rightarrow[(U) / /])$ is reduced to $(\rightarrow[U])$. In particular, the analogy between $(\rightarrow[*])$ and $(\rightarrow[(*) / /])$ is exciting, because the rule $(\rightarrow[*])$ is the dynamic logical counterpart of celebrated Floyd's invariant assertion method and the rule $(\rightarrow[(*) / /])$ is its extension to concurrent programs. The rule $(\rightarrow[*])$ can be illustrated by Figure 3.1. Similarly Figures 3.2 and 3.3 depict the rule $(\rightarrow[(*) / /])$ in case of $\beta=a ; b$ and $\beta=(a ; b)^{*}$ respectively.

We now recall the notions of the characteristic $w f f \psi(\Gamma \rightarrow \Delta)$ of a sequent $\Gamma \rightarrow \Delta$ and of the characteristic wff $\psi(X)$ of a finite set $X$ of sequents, which were defined in Nishimura [5] as follows:

(1) $\psi(\Gamma \rightarrow \Delta)=A_{1} \wedge \cdots \wedge A_{n} \wedge \neg B_{1} \wedge \cdots \wedge \neg B_{m}$, where $\Gamma=\left\{A_{1}, \ldots, A_{n}\right\}$ and $\Delta=\left\{B_{1}, \ldots, B_{m}\right\}$.

(2) $\psi(X)=\psi\left(\Gamma_{1} \rightarrow \Delta_{1}\right) \vee \cdots \vee \psi\left(\Gamma_{k} \rightarrow \Delta_{k}\right)$, where $X=\left\{\Gamma_{1} \rightarrow \Delta_{1}, \ldots, \Gamma_{k} \rightarrow \Delta_{k}\right\}$.

We borrow the following lemma from Nishimura [5].

Lemma 3.1. For any sequent $\Gamma, \Pi \rightarrow \Delta, \Sigma$, we have that $\vdash \Gamma, \Pi \rightarrow \Delta, \Sigma$ iff $\vdash \psi(\Gamma \rightarrow \Delta), \Pi \rightarrow \Sigma$.

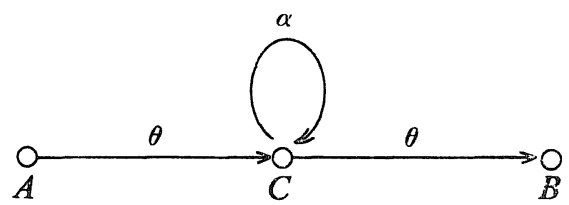

Figure. 3.1

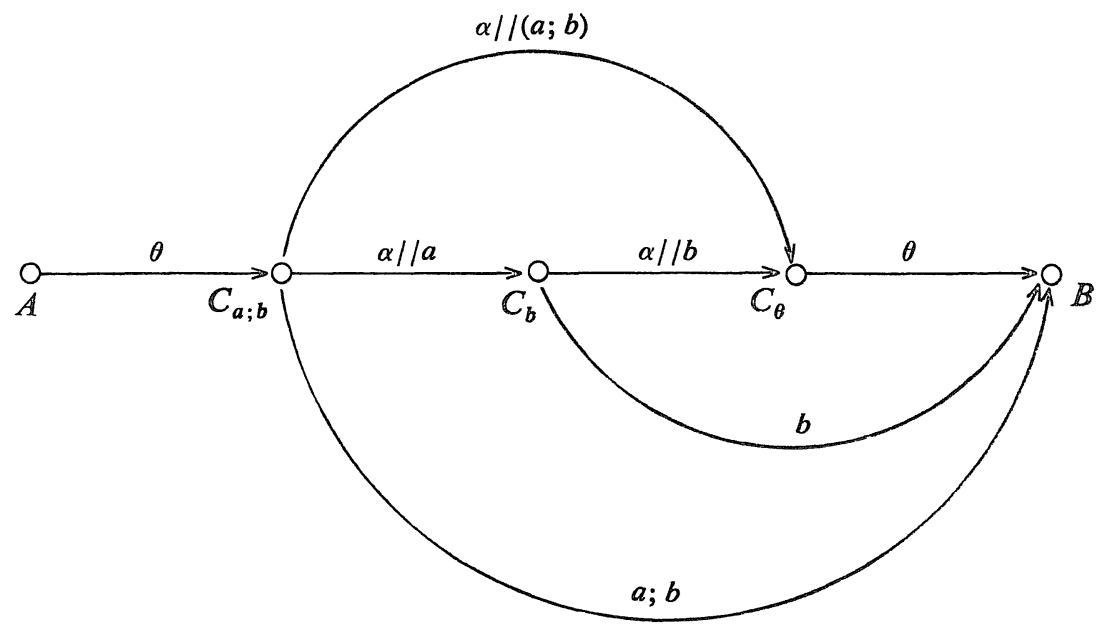

Figure. 3.2 


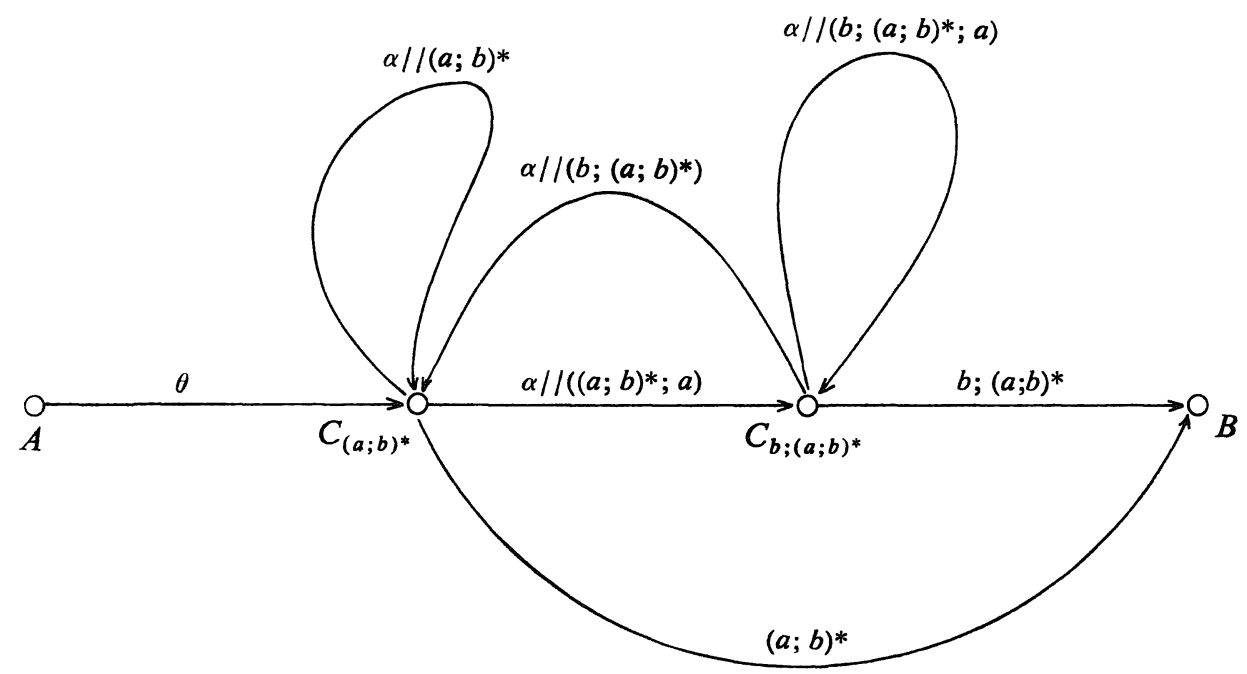

Figure. 3.3

\section{§4. Soundness}

Theorem 4.1 (Soundness Theorem for GCPDL). For any sequent $\Gamma \rightarrow \Delta$, if $\vdash \Gamma \rightarrow \Delta$, then $\vDash \Gamma \rightarrow \Delta$.

Proof. The proof is carried out by induction on the construction of a proof of the given sequent. It follows immediately from the definition that any axiom sequent $A \rightarrow A$ is valid. As for the inference rules, we deal only with $(\rightarrow[(*) / /])$, since other cases are similar or easier. Thus we must consider Figure 4.1.

$$
\begin{aligned}
\frac{A \rightarrow C_{\beta}\left\{C_{\gamma} \rightarrow[\alpha / / \text { init }(i, \gamma)] C_{\text {rest }(i, r)} \mid \gamma \in \operatorname{rest}-\mathrm{cl}(\beta)\right.}{A \rightarrow\left[\alpha^{*} / / \beta\right] B} & \\
\text { and } 0 \leqq i \leqq \mathrm{~d}(\gamma)\}\left\{C_{\gamma} \rightarrow[\gamma] B \mid \gamma \in \operatorname{rest}-\operatorname{cl}(\beta)\right\} & (\rightarrow[(*) / /])
\end{aligned}
$$

Figure. 4.1

By induction hypothesis, the upper sequents are valid. I.e.,
(A)
$\vDash A \longrightarrow C_{\beta}$,
(B)
$\vDash C_{\gamma} \longrightarrow[\alpha / /$ init $(i, \gamma)] C_{\text {rest }(i, \gamma)}$ for
each $\gamma \in \operatorname{rest}-\operatorname{cl}(\beta)$ and each $0 \leqq i \leqq \mathrm{~d}(\gamma)$,
(C)
$\vDash C_{\gamma} \longrightarrow[\gamma] B$ for each $\gamma \in \operatorname{rest}-\operatorname{cl}(\beta)$. 
Assume, for the sake of contradiction, that the lower sequent $A \rightarrow\left[\alpha^{*} / / \beta\right] B$ is realizable. Then there must exist some structure $(W, \rho, \pi)$ and some $w \in W$ such that:

$$
\begin{aligned}
& \pi(A, w)=1, \\
& \pi\left(\left[\alpha^{*} / / \beta\right] B, w\right)=0 .
\end{aligned}
$$

(E) implies that there exists some $h \in \rho_{r}\left(\alpha^{*} / / \beta\right)$ such that:

$$
\begin{aligned}
& \text { is }(h)=w, \\
& \pi(B, \mathrm{fs}(h))=0 .
\end{aligned}
$$

Since $\rho_{r}\left(\alpha^{*} / / \beta\right)=\bigcup_{n=0}^{\infty} \rho_{r}\left(\alpha^{n} / / \beta\right)$, we have $h \in \rho_{r}\left(\alpha^{n} / / \beta\right)$ for some $n \in \mathbb{N}$. Hence there exist some $h_{1}, \ldots, h_{n+1} \in \mathrm{H}_{r}(W)$, some programs $\gamma_{1}, \ldots, \gamma_{n}, \delta_{1}, \ldots, \delta_{n}$ and some $i_{1}, \ldots, i_{n} \in \boldsymbol{N}$ such that :

$$
\begin{array}{ll}
\text { (H) } & h=h_{1}, \ldots ; h_{n+1}, \\
\text { (I) } & h_{j} \in \rho_{r}\left(\alpha / / \gamma_{j}\right) \text { for each } 1 \leqq j \leqq n, \\
\text { (J) } & h_{n+1} \in \rho_{r}\left(\delta_{n}\right), \\
\text { (K) } & \gamma_{1}=\operatorname{init}\left(i_{1}, \beta\right) \text { and } \delta_{1}=\operatorname{rest}\left(i_{1}, \beta\right), \\
\text { (L) } & \gamma_{j}=\operatorname{init}\left(i_{j}, \delta_{j-1}\right) \text { and } \delta_{j}=\operatorname{rest}\left(i_{j}, \delta_{j-1}\right) \text { for each } 2 \leqq j \leqq n .
\end{array}
$$

(A) and (D) imply that:

$$
\pi\left(C_{\beta}, w\right)=1 .
$$

It follows from (H) and (I) that is $\left(h_{1}\right)=w$ and $h_{1} \in \rho_{r}\left(\alpha / / \gamma_{1}\right)$. Hence, by applying (B) to the sequent $C_{\beta} \rightarrow\left[\alpha / / \gamma_{1}\right] C_{\delta_{1}},(\mathrm{M})$ implies that:

$$
\pi\left(C_{\delta_{1}}, \text { fs }\left(h_{1}\right)\right)=1 \text {. }
$$

Similar arguments show that:

$$
\pi\left(C_{\delta_{j}}, \text { fs }\left(h_{j}\right)\right)=1 \text { for each } 2 \leqq j \leqq n .
$$

In particular,

$$
\pi\left(C_{\delta_{n}}, \text { is }\left(h_{n+1}\right)\right)=\pi\left(C_{\delta_{n}}, \text { fs }\left(h_{n}\right)\right)=1 \text {. }
$$

Hence, by applying (C) to the sequent $C_{\delta_{n}} \rightarrow\left[\delta_{n}\right] B,(J)$ and (P) imply that:

$$
\pi(B, \text { fs }(h))=\pi\left(B, \text { fs }\left(h_{n+1}\right)\right)=1 \text {. }
$$

(G) and (Q) contradict, which is the desired conclusion. This completes the proof.

Theorem 4.2 (Consistency of GCPDL). The empty sequent $\rightarrow$ is consistent. 
Proof. Follows from Theorem 4.1.

\section{§5. Completeness}

The main purpose of this section is to establish the semantical completeness of our sequential system $\mathrm{GCPDL}$ with respect to the relational semantics discussed in Section 2. I.e., this section is devoted to the proof of the following theorem.

Theorem 5.1 (Completeness Theorem for GCPDL). Any consistent sequent $\Gamma \rightarrow \Delta$ is realizable.

A finite set $\Phi$ of wffs is called closed if it satisfies the following conditions:

(1) If $(A \wedge B) \in \Phi$, then $A \in \Phi$ and $B \in \Phi$.

(2) If $(A \vee B) \in \Phi$, then $A \in \Phi$ and $B \in \Phi$.

(3) If $\neg A \in \Phi$, then $A \in \Phi$.

(4) If $(A \supset B) \in \Phi$, then $A \in \Phi$ and $B \in \Phi$.

(5) If $[\alpha] A \in \Phi$, then $A \in \Phi$.

(6) If $[\alpha ; \beta] A \in \Phi$, then $[\alpha][\beta] A \in \Phi$.

(7) If $[\alpha \cup \beta] A \in \Phi$, then $[\alpha] A \in \Phi$ and $[\beta] A \in \Phi$.

(8) If $\left[\alpha^{*}\right] A \in \Phi$, then $[\alpha]\left[\alpha^{*}\right] A \in \Phi$.

(9) If $[\alpha / / \beta] A \in \Phi$ and $\alpha$ is an indivisible program, then [init $(i, \beta)][\alpha]$ $[$ rest $(i, \beta)] A \in \Phi$ for any $0 \leqq i \leqq \mathrm{~d}(\beta)$.

(10) If $([(\alpha ; \beta) / / \gamma] A \in \Phi$, then $[\alpha / /$ init $(i, \gamma)][\beta / /$ rest $(i, \gamma)] A \in \Phi$ for any $0 \leqq i \leqq \mathrm{~d}(\gamma)$.

(11) If $[(\alpha \cup \beta) / / \gamma] A \in \Phi$, then $[\alpha / / \gamma] A \in \Phi$ and $[\beta / / \gamma] A \in \Phi$.

(12) If $\left[\alpha^{*} / / \beta\right] A \in \Phi$, then $[\beta] A \in \Phi$ and $[\alpha / / \operatorname{init}(i, \beta)]\left[\alpha^{*} / / \operatorname{rest}(i, \beta)\right] A \in \Phi$ for any $0 \leqq i \leqq \mathrm{~d}(\beta)$.

(13) If $[(\alpha / / \beta) / / \gamma] A \in \Phi$, then $[\alpha / /(\beta / / \gamma)] A \in \Phi$.

In the rest of this section we fix such a closed set, say, $\Phi$. A sequent $\Gamma \rightarrow \Delta$ is called $\Phi$-saturated if it satisfies the following conditions:

(1) $\Gamma \rightarrow \Delta$ is consistent.

(2) $\Gamma \cup \Delta=\Phi$.

It is easy to see that for any $\Phi$-saturated sequent $\Gamma \rightarrow \Delta, \Gamma \cap \Delta=\varnothing$. We borrow the following from Nishimura [5].

Lemma 5.2. Any consistent sequent $\Gamma \rightarrow \Delta$ can be extended to some consistent sequent $\widetilde{\Gamma} \rightarrow \tilde{\Delta}$ such that $\Phi \leqq \tilde{\Gamma} \cup \tilde{\Delta}$. 
Corollary 5.3. Any consistent sequent $\Gamma \rightarrow \Delta$, where $\Gamma \cup \Delta \subseteq \Phi$, can be extended to some $\Phi$-saturated sequent.

Now we define the $\Phi$-canonical structure $\mathrm{S}(\Phi)=(W, \rho, \pi)$ as follows:

(1) $W=\{\Gamma \rightarrow \Delta \mid \Gamma \rightarrow \Delta$ is $\Phi$-saturated $\}$.

(2) $\rho(a)=\left\{\left(\Gamma_{1} \rightarrow \Delta_{1}, \Gamma_{2} \rightarrow \Delta_{2}\right) \in W \times W \mid\left\{A \mid[a] A \in \Gamma_{1}\right\} \cong \Gamma_{2}\right\}$ for any atomic program $a$.

(3) For any atomic formula $p, \pi(p, \Gamma \rightarrow \Delta)=1$ iff $p \in \Gamma$.

The rest of this section is devoted almost completely to the proof of the following theorem, for which several auxiliary notions and lemmas are in order and from which Theorem 5.1 follows at once.

Theorem 5.4 (Fundamental Theorem of $\mathrm{S}(\Phi)$ ). For any wff $A \in \Phi$ and any sequent $\Gamma \rightarrow \Delta$ of $W, \pi(A, \Gamma \rightarrow \Delta)=1$ if $A \in \Gamma$ and $\pi(A, \Gamma \rightarrow \Delta)=0$ if $A \in \Delta$. There fore $\pi(\Gamma \rightarrow \Delta, \Gamma \rightarrow \Delta)=1$.

We define two functions $\omega_{1}$ and $\omega_{2}$ from the set of all programs to the set $\mathbb{N}$ of all non-negative integers as follows:

(1) $\omega_{1}(\alpha)=1$ for any indivisible program $\alpha$.

(2) $\omega_{1}(\alpha ; \beta)=\omega_{1}(\alpha \cup \beta)=\omega_{1}(\alpha)+\omega_{1}(\beta)+1$.

(3) $\omega_{1}\left(\alpha^{*}\right)=\omega_{1}(\alpha / / \beta)=\omega_{1}(\alpha)+1$.

(4) $\omega_{2}(\alpha)=0$ for any indivisible program $\alpha$.

(5) $\omega_{2}(\alpha ; \beta)=\omega_{2}(\alpha \cup \beta)=\max \left\{\omega_{2}(\alpha), \omega_{2}(\beta)\right\}$.

(6) $\omega_{2}\left(\alpha^{*}\right)=\omega_{2}(\alpha)$.

(7) $\omega_{2}(\alpha / / \beta)=\omega_{2}(\alpha)+\omega_{2}(\beta)+1$.

We denote by $<$ the usual lexicographic order on $\mathbb{N} \times \mathbb{N}$. I.e., for any $\left(i_{1}, i_{2}\right),\left(j_{1}, j_{2}\right) \in \mathbb{N} \times \mathbb{N},\left(i_{1}, i_{2}\right)<\left(j_{1}, j_{2}\right)$ iff one of the following conditions holds:

(1) $i_{2}<j_{2}$

(2) $i_{2}=j_{2}$ and $i_{1}<j_{1}$.

We decree that $\Omega(\alpha)=\left(\omega_{1}(\alpha), \omega_{2}(\alpha)\right)$ for any program $\alpha$. We borrow the following from Nishimura [7].

\section{Lemma 5.5.}

(1) $\Omega(\alpha)<\Omega(\alpha ; \beta)$ and $\Omega(\beta)<\Omega(\alpha ; \beta)$.

(2) $\Omega(\alpha)<\Omega(\alpha \cup \beta)$ and $\Omega(\beta)<\Omega(\alpha \cup \beta)$.

(3) $\Omega(\alpha)<\Omega\left(\alpha^{*}\right)$.

(4) $\Omega(\alpha)<\Omega(\alpha / / \beta), \Omega($ init $(i, \beta))<\Omega(\alpha / / \beta)$ and $\Omega(\operatorname{rest}(i, \beta))<\Omega(\alpha / / \beta)$ for any $i \in \mathbb{N}$. 
(5) $\Omega(\alpha / /$ init $(i, \gamma))<\Omega((\alpha ; \beta) / / \gamma)$ and $\Omega(\beta / /$ rest $(i, \gamma))<\Omega((\alpha ; \beta) / / \gamma)$ for any $i \in \mathbf{N}$.

(6) $\Omega(\alpha / / \gamma)<\Omega((\alpha \cup \beta) / / \gamma)$ and $\Omega(\beta / / \gamma)<\Omega((\alpha \cup \beta) / / \gamma)$.

(7) $\Omega(\alpha / / \operatorname{init}(i, \gamma))<\Omega\left(\alpha^{*} / / \beta\right)$ and $\Omega(\gamma)<\Omega\left(\alpha^{*} / / \beta\right)$ for any $\gamma \in \operatorname{rest}-\operatorname{cl}(\beta)$ and any $0 \leqq i \leqq \mathrm{~d}(\gamma)$.

(8) $\Omega(\alpha / /(\beta / / \gamma))<\Omega((\alpha / / \beta) / / \gamma)$.

For any $Y \subseteq W$ and any program $\alpha$, the gravitated set of $Y$ by $\alpha$, denoted by $\mathrm{GS}(\alpha, Y)$, is defined as follows:

GS $(\alpha, Y)=\left\{w \in W \mid\right.$ for any $h \in \rho_{r}(\alpha), \quad$ if is $(h)=w, \quad$ then fs $\left.(h) \in Y\right\}$.

For any $X, Y \subseteq W$ and any program $\alpha$, we say that $Y$ is closed under $\alpha$ relative to $X$ (notation: $\mathrm{cl}(\alpha, X, Y)$ ) if $X \subseteq \mathrm{GS}(\alpha, Y)$. In the rest of this section we will often identify a set $\{w\}$ consisting of a single element $w$ with $w$ itself.

\section{Lemma 5.6.}

(1) $\operatorname{cl}(\theta, X, Y)$ iff $X \subseteq Y$.

(2) $\operatorname{cl}(\alpha ; \beta, X, Y)$ iff $\operatorname{cl}(\alpha, X, \mathrm{GS}(\beta, Y))$.

(3) $\operatorname{cl}(\alpha \cup \beta, X, Y)$ iff $\operatorname{cl}(\alpha, X, Y)$ and $\operatorname{cl}(\beta, X, Y)$.

(4) $\operatorname{cl}\left(\alpha, \operatorname{GS}\left(\alpha^{*}, X\right), \operatorname{GS}\left(\alpha^{*}, X\right)\right)$.

(5) $\mathrm{GS}\left(\alpha^{*}, X\right) \subseteq X$.

(6) Let $\alpha$ be an indivisible program. Then $\operatorname{cl}(\alpha / / \beta, X, Y)$ iff $\mathrm{cl}$ (init $(i, \beta)$, $X, \operatorname{GS}(\alpha ;$ rest $(i, \beta), Y))$ for any $0 \leqq i \leqq \mathrm{~d}(\beta)$.

(7) $\operatorname{cl}(\alpha, \operatorname{GS}(\alpha ; \beta, X), \operatorname{GS}(\beta, X))$.

(8) $\operatorname{cl}((\alpha ; \beta) / / \gamma, X, Y)$ iff $\operatorname{cl}(\alpha / /$ init $(i, \gamma), X, \operatorname{GS}(\beta / /$ rest $(i, \gamma), Y))$ for any $0 \leqq i \leqq \mathrm{~d}(\gamma)$.

(9) $\operatorname{cl}((\alpha \cup \beta) / / \gamma, X, Y)$ iff $\operatorname{cl}(\alpha / / \gamma, X, Y)$ and $\operatorname{cl}(\beta / / \gamma, X, Y)$.

(10) $\mathrm{cl}\left(\alpha / / \operatorname{init}(i, \beta), \mathrm{GS}\left(\alpha^{*} / / \beta, Y\right), \mathrm{GS}\left(\alpha^{*} / / \operatorname{rest}(i, \beta), Y\right)\right)$ for any $0 \leqq i$ $\leqq \mathrm{d}(\beta)$.

(11) $\operatorname{cl}\left(\beta, \mathrm{GS}\left(\alpha^{*} / / \beta, X\right), X\right)$.

(12) $\operatorname{cl}((\alpha / / \beta) / / \gamma, X, Y)$ iff $\operatorname{cl}(\alpha / /(\beta / / \gamma), X, Y)$.

Lemma 5.7. For any $X, Y \subseteq W$ and any program $\alpha$, if $\operatorname{cl}(\alpha, X, Y)$, then $\vdash \psi(X) \rightarrow[\alpha] \psi(Y)$.

Proof. By induction on $\Omega(\alpha)$. Lemma 5.5 is implicit in the following.

(1) $\alpha=\theta:$ By (1) of Lemma 5.6, 
Hence, by applying rule $(\rightarrow[\theta])$ to $(\mathrm{A})$, we obtain

$$
\vdash \psi(X) \longrightarrow[\theta] \psi(Y) \text {. }
$$

(2) $\alpha$ is an atomic program, say, a: Let $X=\left\{\Gamma_{i} \rightarrow \Delta_{i} \mid 1 \leqq i \leqq n\right\}$. We assume, for the sake of simplicity, that $n=2$. Then it is easy to see that

$$
\vdash \psi(X) \longrightarrow \psi\left(\Gamma_{1} \rightarrow \Delta_{1}\right), \psi\left(\Gamma_{2} \rightarrow \Delta_{2}\right) .
$$

Suppose, for the sake of contradiction, that the sequent $\psi\left(\Gamma_{1} \rightarrow \Delta_{1}\right) \rightarrow[a] \psi(Y)$ is consistent, which implies, by Lemma 3.1, that the sequent $\Gamma_{1} \rightarrow \Delta_{1},[a] \psi(Y)$ is also consistent. So the sequent $\left\{A \mid[a] A \in \Gamma_{1}\right\} \rightarrow \psi(Y)$ is also consistent, for otherwise $\Gamma_{1} \rightarrow \Delta_{1}$, [a] $\psi(Y)$ would be provable by rules $(\rightarrow[])$ and (extension). By Lemma 5.2, the sequent $\left\{A \mid[a] A \in \Gamma_{1}\right\} \rightarrow \psi(Y)$ can be extended to some consistent sequent $\tilde{\Gamma} \rightarrow \tilde{\Delta}$ such that $\Phi \leqq \tilde{\Gamma} \cup \tilde{\Delta}$. Then it is easy to see that $\left(\Gamma_{1} \rightarrow \Delta_{1}, \tilde{\Gamma} \cap \Phi \rightarrow \tilde{\Delta} \cap \Phi\right) \in \pi(a)$. Since $\operatorname{cl}\left(a, \Gamma_{1} \rightarrow \Delta_{1}, Y\right)$ by assumption, $(\tilde{\Gamma} \cap \Phi$ $\rightarrow \tilde{\Delta} \cap \Phi) \in Y$. So

$$
\vdash \psi(\tilde{\Gamma} \cap \Phi \rightarrow \tilde{\Delta} \cap \Phi) \longrightarrow \psi(Y)
$$

This implies, by Lemma 3.1, that

$$
\vdash \tilde{\Gamma} \cap \Phi \longrightarrow \tilde{\Delta} \cap \Phi, \psi(Y) .
$$

This contradicts the assumption that the sequent $\tilde{\Gamma} \rightarrow \tilde{\Delta}$ is consistent and $\psi(Y) \in \tilde{\Delta}$. Thus we can conclude that

$$
\vdash \psi\left(\Gamma_{1} \rightarrow \Delta_{1}\right) \longrightarrow[a] \psi(Y) .
$$

A similar argument shows that

$$
\vdash \psi\left(\Gamma_{2} \rightarrow \Delta_{2}\right) \longrightarrow[a] \psi(Y) .
$$

By using rule (cut) twicc, we can deduce from (A), (D) and (E) that

$$
\vdash \psi(X) \longrightarrow[a] \psi(Y) .
$$

(3) $\alpha=\beta ; \gamma$ : By (2) of Lemma 5.6, we have $\operatorname{cl}(\beta, X, \operatorname{GS}(\gamma, Y))$. Hence by induction hypothesis,

$$
\vdash \psi(X) \longrightarrow[\beta] \psi(\mathrm{GS}(\gamma, Y)) .
$$

Since $\mathrm{cl}(\gamma, \mathrm{GS}(\gamma, Y), Y)$, we have by induction hypothesis that

$$
\vdash \psi(\mathrm{GS}(\gamma, Y)) \longrightarrow[\gamma] \psi(Y)
$$

By applying $(\rightarrow[])$ to $(B)$, we have 


$$
\vdash[\beta] \psi(\mathrm{GS}(\gamma, Y)) \longrightarrow[\beta][\gamma] \psi(Y) .
$$

By using (cut), we have from (A) and (C) that

$$
\vdash \psi(X) \longrightarrow[\beta][\gamma] \psi(Y) .
$$

By applying $(\rightarrow[;])$ to $(D)$, we have

$$
\vdash \psi(X) \longrightarrow[\beta ; \gamma] \psi(Y) .
$$

(4) $\alpha=\beta \cup \gamma$ : By (3) of Lemma 5.6, $\operatorname{cl}(\beta, X, Y)$ and $\operatorname{cl}(\gamma, X, Y)$. Hence by induction hypothesis,$$
\vdash \psi(X) \longrightarrow[\beta] \psi(Y)
$$$$
\vdash \psi(X) \longrightarrow[\gamma] \psi(Y) .
$$

By applying rule $(\rightarrow[\mathrm{U}])$ to $(\mathrm{A})$ and $(\mathrm{B})$, we have

$$
\vdash \psi(X) \longrightarrow[\beta \cup \gamma] \psi(Y) .
$$

(5) $\alpha=\beta^{*}$ : Since $\operatorname{cl}\left(\beta^{*}, X, Y\right)$ by assumption, we have $X \cong \mathrm{GS}\left(\beta^{*}, Y\right)$. Hence

$$
\vdash \psi(X) \longrightarrow \psi\left(\mathrm{GS}\left(\beta^{*}, Y\right)\right) .
$$

By (4) of Lemma 5.6, $\mathrm{cl}\left(\alpha, \mathrm{GS}\left(\alpha^{*}, Y\right), \mathrm{GS}\left(\alpha^{*}, Y\right)\right)$, which implies by induction hypothesis that

$$
\vdash \psi\left(\mathrm{GS}\left(\beta^{*}, Y\right)\right) \longrightarrow[\beta] \psi\left(\mathrm{GS}\left(\beta^{*}, Y\right)\right) .
$$

By (5) of Lemma 5.6, GS $\left(\beta^{*}, Y\right) \subseteq Y$, which implies that

$$
\vdash \psi\left(\mathrm{GS}\left(\beta^{*}, Y\right)\right) \longrightarrow \psi(Y) \text {. }
$$

By applying rule $(\rightarrow[*])$ to $(\mathrm{A}),(\mathrm{B})$ and $(\mathrm{C})$, we have

$$
\vdash \psi(X) \longrightarrow\left[\beta^{*}\right] \psi(Y) .
$$

(6) $\alpha=\beta / / \gamma$ and $\beta$ is an indivisible program: By (6) of Lemma 5.6,

$$
\mathrm{cl}(\text { init }(i, \gamma), X, \mathrm{GS}(\beta ; \text { rest }(i, \gamma), Y)), \text { for any } 0 \leqq i \leqq \mathrm{~d}(\gamma)
$$

which implies by induction hypothesis that

(A) $\vdash \psi(X) \longrightarrow[$ init $(i, \gamma)] \psi(\mathrm{GS}(\beta$; rest $(i, \gamma), Y))$ for any $\gamma \leqq i \leqq \mathrm{~d}(\gamma)$.

By (7) of Lemma 5.6,

$$
\operatorname{cl}(\beta, \mathrm{GS}(\beta ; \text { rest }(i, \gamma), Y), \mathrm{GS}(\text { rest }(i, \gamma), Y)) .
$$

Hence by induction hypothesis, 
(B) $\quad \vdash \psi(\mathrm{GS}(\beta ; \operatorname{rest}(i, \gamma), Y)) \longrightarrow[\beta] \psi(\mathrm{GS}(\operatorname{rest}(i, \gamma), Y))$ for any $0 \leqq i \leqq \mathrm{~d}(\gamma)$. Since $\mathrm{cl}(\operatorname{rest}(i, \gamma)$, GS (rest $(i, \gamma), Y), Y)$, we have by induction hypothesis that (C) $\quad \vdash \psi(\mathrm{GS}(\operatorname{rest}(i, \gamma), Y)) \longrightarrow[\operatorname{rest}(i, \gamma)] \psi(Y)$ for any $0 \leqq i \leqq \mathrm{~d}(\gamma)$. By using rules (cut) and $(\rightarrow[\mathrm{]})$, we can deduce from $(\mathrm{A}),(\mathrm{B})$ and $(\mathrm{C})$ that (D) $\quad \vdash \psi(X) \longrightarrow[\operatorname{init}(i, \gamma)][\beta][\operatorname{rest}(i, \gamma)] \psi(Y)$ for any $0 \leqq i \leqq \mathrm{~d}(\gamma)$. By applying rule $(\rightarrow[/ /])$ to $(D)$, we have

$$
\vdash \psi(X) \longrightarrow[\beta / / \gamma] \psi(Y)
$$

(7) $\alpha=(\beta ; \gamma) / / \delta$ : By (8) of Lemma 5.6, $\mathrm{cl}(\beta / /$ init $(i, \delta), X, \mathrm{GS}(\gamma / /$ rest $(i, \delta), Y)$ ) for any $0 \leqq i \leqq \mathrm{~d}(\delta)$. Hence by induction hypothesis,

(A) $\vdash \psi(X) \longrightarrow[\beta / / \operatorname{init}(i, \delta)] \psi(\mathrm{GS}(\gamma / / \operatorname{rest}(i, \delta), Y))$ for any $0 \leqq i \leqq \mathrm{~d}(\delta)$.

Since $\operatorname{cl}(\gamma / /$ rest $(i, \delta), \operatorname{GS}(\gamma / /$ rest $(i, \delta), Y), Y)$, we have by induction hypothesis that

(B) $\quad \vdash \psi(\mathrm{GS}(\gamma / / \operatorname{rest}(i, \delta), Y)) \longrightarrow[\gamma / / \operatorname{rest}(i, \delta)] \psi(Y)$ for any $0 \leqq i \leqq \mathrm{~d}(\delta)$.

By using rules (cut) and $(\rightarrow[])$, we can deduce from $(A)$ and $(B)$ that

(C) $\quad \vdash \psi(X) \longrightarrow[\beta / / \operatorname{init}(i, \delta)][\gamma / / \operatorname{rest}(i, \delta)] \psi(Y)$ for any $0 \leqq i \leqq \mathrm{~d}(\delta)$.

By applying rule $(\rightarrow[(;) / /])$ to $(\mathrm{C})$, we have

$$
\vdash \psi(X) \longrightarrow[(\beta ; \gamma) / / \delta] \psi(Y) .
$$

(8) $\alpha=(\beta \cup \gamma) / / \delta$ : By (9) of Lemma 5.6, we have $\operatorname{cl}(\beta / / \delta, X, Y)$ and $\operatorname{cl}(\gamma / / \delta, X, Y)$. Hence by induction hypothesis,

$$
\begin{aligned}
& \vdash \psi(X) \longrightarrow[\beta / / \delta] \psi(Y) \\
& \vdash \psi(X) \longrightarrow[\gamma / / \delta] \psi(Y) .
\end{aligned}
$$

By applying rule $(\rightarrow[(\mathrm{U}) / /])$ to $(\mathrm{A})$ and $(\mathrm{B})$, we have

$$
\vdash \psi(X) \longrightarrow[(\beta \cup \gamma) / / \delta] \psi(Y) .
$$

(9) $\alpha=\beta^{*} / / \gamma$ : Since $\operatorname{cl}\left(\beta^{*} / / \gamma, X, Y\right)$ by assumption, we have $X \subseteq$ $\operatorname{GS}\left(\beta^{*} / / \gamma, Y\right)$. Hence

$$
\vdash \psi(X) \longrightarrow \psi\left(\mathrm{GS}\left(\beta^{*} / / \gamma, Y\right)\right)
$$

By (10) of Lemma 5.6, $\mathrm{cl}\left(\beta / /\right.$ init $(i, \delta), \mathrm{GS}\left(\beta^{*} / / \delta, Y\right), \mathrm{GS}\left(\beta^{*} / /\right.$ rest $\left.\left.(i, \delta), Y\right)\right)$ for any $\delta \in \operatorname{rest}-\mathrm{cl}(\gamma)$ and $0 \leqq i \leqq \mathrm{~d}(\delta)$. Hence by induction hypothesis, 
(B) $\quad \vdash \psi\left(\mathrm{GS}\left(\beta^{*} / / \delta, Y\right)\right) \longrightarrow[\beta / /$ init $(i, \delta)] \psi\left(\mathrm{GS}\left(\beta^{*} / /\right.\right.$ rest $\left.\left.(i, \delta), Y\right)\right)$ for any $\delta \in \operatorname{rest}-\operatorname{cl}(\gamma)$ and $0 \leqq i \leqq \mathrm{~d}(\delta)$.

By (11) of Lemma 5.6, $\operatorname{cl}\left(\delta, \operatorname{GS}\left(\beta^{*} / / \delta, Y\right), Y\right)$ for any $\delta \in \operatorname{rest}-\operatorname{cl}(\gamma)$. Hence by induction hypothesis,

$$
\vdash \psi\left(\mathrm{GS}\left(\beta^{*} / / \delta, Y\right)\right) \longrightarrow[\delta] \psi(Y) \text { for any } \delta \in \operatorname{rest}-\operatorname{cl}(\gamma) .
$$

By applying rule $(\rightarrow[(*) / /])$ to $(\mathrm{A}),(\mathrm{B})$ and $(\mathrm{C})$, we have

$$
\vdash \psi(X) \longrightarrow\left[\beta^{*} / / \gamma\right] \psi(Y) .
$$

(10) $\alpha=(\beta / / \gamma) / / \delta$ : By (12) of Lemma 5.6, $\operatorname{cl}(\beta / /(\gamma / / \delta), X, Y)$. Hence by induction hypothesis,

$$
\vdash \psi(X) \longrightarrow[\beta / /(\gamma / / \delta)] \psi(Y) .
$$

By applying rule $(\rightarrow[(/ /) / /])$ to $(\mathrm{A})$, we have

$$
\vdash \psi(X) \longrightarrow[(\beta / / \gamma) / / \delta] \psi(Y) .
$$

Lemma 5.8. For any wff $A$, any program $\alpha$ and any sequent $\Gamma \rightarrow \Delta$ of $W$, if $[\alpha] A \in \Delta$, then there exist a sequent $\Gamma^{\prime} \rightarrow \Delta^{\prime}$ of $w$ and a path $h \in \rho_{r}(\alpha)$ such that is $(h)=\Gamma \rightarrow \Delta$ fs $(h)=\Gamma^{\prime} \rightarrow \Delta^{\prime}$ and $A \in \Delta^{\prime}$.

Proof. Let $X=\{(\Pi-\Sigma) \in W \mid A \in \Pi\}$ suppose, for the sake of contradiction, that $\operatorname{cl}(\alpha, \Gamma \rightarrow \Delta, X)$. Then by Lemma 5.7,

$$
\vdash \psi(\Gamma \rightarrow \Delta) \longrightarrow[\alpha] \psi(X) .
$$

It follows from the definition of $X$ that

$$
\vdash \psi(X) \longrightarrow A \text {. }
$$

By using rules (cut) and $(\rightarrow[])$, we can deduce from (A) and (B) that

$$
\vdash \psi(\Gamma \rightarrow \Delta) \longrightarrow[\alpha] A .
$$

By Lemma 3.1, it follows from (C) that

$$
\vdash \Gamma \longrightarrow \Delta,[\alpha] A,
$$

which contradicts the assumption that the sequent $\Gamma \rightarrow \Delta$ is consistent and $[\alpha] A \in \Delta$. This completes the proof.

Lemma 5.9. For any wff $A$, any program $\alpha$, any path $h \in \rho_{r}(\alpha)$ and any sequents $\Gamma \rightarrow \Delta$ and $\Gamma^{\prime} \rightarrow \Delta^{\prime}$ of $W$, if is $(h)=\Gamma \rightarrow \Delta$, fs $(h)=\Gamma^{\prime} \rightarrow \Delta^{\prime}$ and $[\alpha] A \in \Gamma$, then $A \in \Gamma^{\prime}$.

Proof. By induction on $\Omega(\alpha)$. Since the proof is, in a sensc, dual to that 
of Lemma 5.7, we deal only with the case that $\alpha=\beta^{*} / / \gamma$. Since $h \in \rho_{r}\left(\beta^{*} / / \gamma\right)$ and $\rho_{r}\left(\beta^{*} / / \gamma\right)=\bigcup_{n=0}^{\infty} \rho_{r}\left(\beta^{n} / / \gamma\right)$, there exists some $n \in \mathbb{N}$ such that $h \in \rho_{r}\left(\beta^{n} / / \gamma\right)$. Hence there exist some $h_{1}, \ldots, h_{n+1} \in \mathrm{H}_{r}(W)$ some programs $\delta_{1}, \ldots, \delta_{n}, \zeta_{1}, \ldots, \zeta_{n}$ and some $i_{1}, \ldots, i_{n} \in \boldsymbol{N}$ such that

(A) $\quad h=h_{1} ; \ldots ; h_{n+1}$,

(B) $\quad h_{j} \in \rho_{r}\left(\beta / / \zeta_{j}\right)$ for each $1 \leqq j \leqq n$,

(C) $\quad h_{n+1} \in \rho_{r}\left(\zeta_{n}\right)$,

(D) $\delta_{1}=\operatorname{init}\left(i_{1}, \gamma\right)$ and $\zeta_{1}=\operatorname{rest}(i, \gamma)$,

(E) $\quad \delta_{j}=\operatorname{init}\left(i_{j}, \zeta_{j-1}\right)$ and $\zeta_{j}=\operatorname{rest}\left(i_{j}, \zeta_{j-1}\right)$ for each $2 \leqq j \leqq n$.

Let $\Gamma_{j} \rightarrow \Delta_{j}=\mathrm{fs}\left(h_{j}\right)=$ is $\left(h_{j}+1\right)$ for each $1 \leqq j \leqq n$. The sequent $\left[\beta / / \delta_{1}\right]\left[\beta^{*} / / \zeta_{1}\right] A$, $\Gamma \rightarrow \Delta$ is consistent, for otherwise $\left[\beta^{*} / / \gamma\right] A, \Gamma \rightarrow \Delta$ would be provable by rule $([(*) / /] \rightarrow)$. Since the sequent $\Gamma \rightarrow \Delta$ is $\Phi$-saturated, we have $\left[\beta / / \zeta_{1}\right]\left[\beta^{*} / / \zeta_{1}\right]$ $A \in \Gamma$. So by induction hypothesis, (B) implies that $\left[\beta^{*} / / \zeta_{1}\right] A \in \Gamma_{1}$. By repeating this argument, we have

$$
\left[\beta^{*} / / \zeta_{j}\right] A \in \Gamma_{j} \text { for each } 1 \leqq j \leqq n .
$$

In particular, $\left[\beta^{*} / / \zeta_{n}\right] A \in \Gamma_{n}$. The sequent $\left[\zeta_{n}\right] A, \Gamma_{n} \rightarrow \Delta_{n}$ must be consistent, for otherwise the sequent $\left[\beta^{*} / / \zeta_{n}\right], \Gamma_{n} \rightarrow \Delta_{n}$ would be provable by rule $([(*) / /] \rightarrow)$. Since the sequent $\Gamma_{n} \rightarrow \Delta_{n}$ is $\Phi$-saturated, we have $\left[\zeta_{n}\right] A \in \Gamma_{n}$. Hence by induction hypothesis, $A \in \Gamma^{\prime}$, which is the desired result.

Now we are ready to establish Theorem 5.4.

Proof of Theorem 5.4. By induction on the construction of a wff $A \in \Phi$. Use Lemmas 5.8 and 5.9 in dealing with wffs of the form $[\alpha] A$.

\section{References}

[1] Abrahamson, K., Modal logic of concurrent nondeterministic programs, Semantics of concurrent computation, Lecture Notes in Computer Science, 70 (1979), 21-33, Springer, Berlin-Heidelberg-New York.

[2] Eilenberg, S., Automata, Languages, and Machines, vol. A, Academic Press, New York and London, 1974.

[3] Harel, D., Logics of programs: Axiomatics and descriptive power, MIT, dissertation, 1978.

[4] Harel, D., Kozen, D. and Parikh, R., Process logic: Expressiveness, decidability, completeness, Proceedings IEEE Symposium on Foundations of Computer Science, 1980. 
[5] Nishimura, H., Sequential method in propositional dynamic logic, Acta Informatica, 12 (1979), 377-400.

[6] - Descriptively complete process logic, Acta Informatica, 14 (1980), 359-369.

[7] - Arithmetical completeness in first-order dynamic logic for concurrent programs, Publ. RIMS, 17 (1981), 297-309.

[ 8 ] Pratt, V. R., A practical decision method for propositional dynamic logic, Proceedings 10th Annual ACM Symposium on Theory of Computing, 326-337, 1978.

[9] - Process logic, Proceedings 6th Annual ACM Symposium on the Principles of Programming Languages, 1979, 93-100.

[10] - Models of program logics, Proceedings 20th Annual Symposium on Foundations of Computer Science, 1979, 115-122. 